\title{
PI3K/PDK1/Akt pathways and their fundamental role in Gynecological Cancer
}

\author{
Sofoklis Stavros ${ }^{1,2}$, Kalliopi Pappa', Peter Drakakis ${ }^{1,2}$
}

${ }^{1} 1^{\text {st }}$ OB.GYN Department, School of Medicine, National and Kapodistrian University of Athens, Alexandra Hospital, Athens, Greece ${ }^{2}$ Molecular Biology of Reproduction Unit and Recurrent Abortions Unit, Assisted Reproduction Division, $1^{\text {st }} \mathrm{Ob} / G y n$ Dept, Medical School, National and Kapodistrian University of Athens, Greece

\section{Corresponding Author}

Sofoklis Stavros, MD PhD, Obstetrician and Gynecologist, Fellow of the $1^{\text {st }}$ Department of Obstetrics and Gynecology National and Kapodistrian University of Athens, General Hospital of Athens "Alexandra" Loulou and Vasilissis Sofias Ave, 11528 Athens, Greece

tel.: 30 6906725017, e-mail: sfstavrou@yahoo.com

\section{Abstract}

The PI3K/PDK1/Akt pathway can regulate a large variety of cellular processes such as cell growth, proliferation and survival. Moreover, it is one of the most frequently altered networks in cancer though. The dysregulation of these pathways has been reported in several human cancers, as well as in diabetes, neurological diseases and cardiovascular diseases. Cervical carcinogenesis seems to be associated with increased levels of activated AKT kinase. Moreover, PTEN inactivation is the most common genetic alteration in type 1 endometrial cancer. In addition, the PI3K /AKT pathway is related to ovarian carcinomas cell motility and adhesion. This pathway plays an imperative role in cell survival and except for that, it may lead to metastatic and invasive phenotypes of a large variety of malignant cells. A battery of cancerous types has been associated with PI3K/AKT hyperactivation; inhibition of this pathway has been described as a treatment option of malignancy. The role of PI3K-PDK-AKT signaling pathway in gynecological malignancy seems to play a pivotal role, but it necessitates further investigation, which could lead to novel therapeutic strategies of eventual robust advantages to many cancer patients. The aim of that review is to highlight and underline the role of those pathways paving the way of gynecological malignancy.

\section{Introduction}

The PI3K/PDK1/Akt pathway can regulate a large variety of cellular processes such as cell growth, proliferation and survival ${ }^{1}$. Moreover, it is one of the most frequently altered networks in cancer ${ }^{2}$. The dysregulation of these pathways has been reported in several human cancers, as well as in diabetes, neurological diseases and cardiovascular diseases. The different components which take part in this pathway consist important and attractive targets for the therapy ${ }^{1}$. 


\section{Discussion}

Mirza et al, in a recent study suggests that PI3K/ PDK/AKT and RAF/MEK/ERK pathways may collaborate to advocate G0-G1-S phase cell cycle progression in normal and cancer cells alike; this cooperation between them can promote the multiplication of normal cells as well as the abnormal proliferation of cancer cells ${ }^{3}$.

Activation of the Akt/PI3K pathway can be occurred via binding of ligands, i.e. cytocines, growth factors or insulin, to cell membrane receptor tyrosine kinases, leading to autophosphorylation of their intracellular domain. This allows for conformational changes and subsequent phosphatidylinositol 3-kinase (PI3K) activation, a heterodimeric protein consisting of a catalytic (p110) and regulatory (p85) subunit. Based on the differences in structure, PI3K has been divided into three classes (I, II and III); among them, class I plays a crucial role in the onset of many cancers, the most thoroughly researched. PI3K leads to transformation of phosphatidylinositol 4, 5-bisphosphate (PIP2) to phosphatidylinositol 3, 4, 5-trisphosphate (PIP3), which in turn, via phosphoinositide-dependent kinase 1 (PDK1), allows for phosphorylation and activation of the Akt protein. PTEN and INPP4B exert a contradictory effect in cells, transforming PIP3 to PIP2 and PIP2 to PIP1, respectively4.

The family of phosphatidylinositol 3- kinase was first described in the mid-1980s and until now, it plays a pivotal role in cellular functions such as multiplication and survival ${ }^{5}$. PI3k proteins are heterodimers containing two subunits in their form; the catalytic $\mathrm{p} 110$ subunit and the regulatory p85 subunit ${ }^{6}$. Three isoforms of p110 have been described: pd110 $\alpha, \mathrm{p} 110 \beta$ and $\mathrm{p} 110 \delta$ consisting all products of separate genes (PIK3CA, PIK3CB and PIK3CD, respectively) ${ }^{5}$. Regarding subunits p85, five categories have been revealed, including $\mathrm{p} 85 \alpha$, p85 $\beta$, p $55 \alpha, p 55 \gamma$ and p50 $\alpha^{7}$. Based on their lipid substratum, function and structure PI3K proteins divided into three categories; class I, II and class III ${ }^{5}$. Class IA enzymes are activated mostly by tyrosine kinase receptors and special oncogenes such as RAS (rat sarcoma), while Class IB is exclusively activated by $\mathrm{G}$ protein - coupled receptors ${ }^{5}$.

The class IA PI3-K signaling cataract plays a major role, connecting multiple receptors to many processes such as cell cycle, cell survival protein synthesis, metabolism, growth and angiogenesis through other protein families including SGK and $\mathrm{AKT}^{7}$.

Recruitment of PDK1 and AKT plays an important role in interaction of the two kinases. PDK can phosphorylate and fully activate AKT at threonine 308 after the phosphorylation of AKT at the serine 473 site by MTORC2 (mammalian target of rapamycin complex 2) $)^{5,7}$.

Desregulations of the components of PI3K pathway, such as PI3K, PTEN (phosphatase and tensin homolog) and AKT, seem to play a pivotal role in human cancer and especially occur in PIK3CA gene and tumor suppressor PTEN7.

In addition, abnormal mutations in PI3K pathway can also be presented in cancers after receptor tyrosine kinases mutation ${ }^{5}$.

Mutations in AKT family genes have also been presented in human cancers; a single amino acid substitution, E17K, in the plecstrin homology domain may also lead to constitutive recruitment of AKT to the membrane ${ }^{5}$.

AKT2 was amplified and over expressed in a subset of ovarian malignancies; more specifically two out of eight ovarian tumor cell lines and two of 15 primary ovarian malignancies have been reported based on the above alterations ${ }^{8}$. In addition, AKT2 has been described that can be amplified in non-Hodgkin lymphomas ${ }^{9}$. Furthermore, amplification and /or overexpression of AKT2 has been presented in 10$20 \%$ of primary pancreatic malignancies and cell lines of pancreatic tumours ${ }^{10,11,12}$. Moreover almost $40 \%$ of hepatocellular carcinomas are related to an 
increased expression of AKT2, where the overexpression of AKT2 plays an independent prognostic role ${ }^{13}$.

Instead AKT2, AKT1 is amplified not so frequently based on bibliography. Staal and al have shown that AKT1 amplification is associated with gastric carcinomas ${ }^{14}$, while in a more recent investigation of 103 glial tumors, AKT1 amplification and overexpression were revealed in a single case of gliosarcoma ${ }^{15}$.

AKT3 high level amplification has not been reported in human malignancies. Small increases in a number of AKT3 copies are expected in a subset of tumors. Estrogen receptor -negative breast tumors have been shown an upregulation of AKT3 m RNA ${ }^{16}$.

Advanced disease and poor prognosis are related to AKT activation in some types of malignancy. Recent study has shown that approximately $40 \%$ of ovarian and breast malignancies and more than the half of prostate malignant lesions exhibit increased AKT1 kinase activity; the most imperative result of this study is that almost the $80 \%$ of these malignancies were stage III/IV cancers and grade 3 as well ${ }^{17}$. Other studies have revealed that activation of AKT2 kinase was reported in 30 to $40 \%$ of ovarian and pancreatic malignant lesions ${ }^{18,19}$.

The PIP3 as a PI3K product is an anchor for the further recruitment of downstream effectors such as PDK1 and AKT. PIP3 is a crucial component of PI3K-mediated oncogenesis. The activation of PI3K or inactivation of PTEN (phosphatase and tensin homolog) can trigger increased levels of PIP3.This last finding is frequently revealed in tumor cells ${ }^{1}$.

\section{Pathways and Cervical carcinoma}

Cervical carcinoma is the second most common malignancy of female reproductive tract and ranks second among female deaths caused by cancers ${ }^{20}$. In a recent research, Bertelsen et al, showed that cervical carcinogenesis seems to be associated with increased levels of activated AKT kinase; this can be explained by PIK3CA amplification, whereas PTEN mutation may not play such an important role ${ }^{21}$.

Furthermore, PTEN's loss of function may lead to activation of this pathway via PIP3 accumulation. Inactivating mutations of the tumor suppressor gene, PTEN, have been detected in endometrial and ovarian cancer alike. PTEN is an inhibitor of PI3K/ AKT signaling as well as it controls the rate of cell division and promote apoptosis. Thus, the fundamental in vivo role of PTEN is to inhibit the PI3K-AKT pathway. Mutation of PTEN can disable this inhibitory function, thus inducing the antiapoptotic pathway, which is believed to be the key to endometrial carcinogenesis ${ }^{22}$.

\section{Pathways and Endometrial carcinoma}

PTEN inactivation is the most common genetic alteration in type 1 endometrial cancer. The highest rates $(83 \%)$ occur in sporadic cases with a simultanusly or a prior premalignant lesion. Moreover, the pivotal role of PTEN in endometrial cancer type 1 has been reported in PTEN knockout mice with a percentage of $20 \%$ endometrial cancer development. Opposite to type 1 endometrial malignancy, loss of PTEN isn't reported in type 2 endometrial tumors. Type I endometrial carcinogenesis has been related with a variety of hormonal mechanisms such as the influence of estrogens; earlier endometrial stages, precancer lesions and endometrial intraepithelial neoplasia (EIN) have been shown to maintain high levels of nuclear estrogen and progesterone receptors and under conditions of estrogen stimulation it is hypothesized that PTEN defective cells would have a selective proliferative advantage since PTEN would not carry out its normal role to limit the rate of cell division ${ }^{23}$.

\section{Pathways and Ovarian carcinoma}

Among women in Europe and United States, ovarian carcinoma consists the fourth pivotal causal factor of cancer mortality. Its early diagnosis is difficult because lacks specific clinical symptoms of this entity. 
On the other hand, its late detection leads to poor survival rates for the diagnosed patients. Numerous causal factors have been associated with ovarian carcinomas development such as the age, the hormonal profile (early or late menopausal women, infertility, endometriosis), genetic factors and environmental factors (smoking, diet, obesity) ${ }^{24}$.

The PI3K /AKT pathway is related to ovarian carcinomas cell motility and adhesion. This pathway plays an important role in cell survival and except for that, it may lead to metastatic and invasive phenotypes of a large variety of malignant cells. A battery of cancerous types has been associated with PI3K/AKT hyperactivation; so, inhibition of this pathway can be considered as a treatment option of malignancy ${ }^{24}$.

Overexpression and/or activation of the PI3K/Akt survival pathway have been revealed to carcinogenesis of numerous tissue types. In a recent study, $\mathrm{Wu}$ et al showed that the phosphorylated AKT levels are increased in chemo resistant ovarian malignancy. Hence, therapy including Akt inhibitor leads to increased sensitivity to anticancer drugs. In addition, this study further showed that COL11A1 (collagen type XI alpha 1) may lead to increased phosphorylated Akt in chemo resistant ovarian cancer cells via stabilizing PDK1 protein. Moreover, Wu et al, revealed that phosphorylated Akt levels in chemo resistant ovarian carcinomas are increased by COL11A1 due to enhanced binding activity between PDK1 and COL11A1. Over the course of this study, an important result has been revealed; the overexpression of COL11A1 leads to chemoresistance, possibly by binding to PDK1-akt, and subsequently preventing PDK1 degradation. This binding may prevent cisplatin- and paclitaxel-induced PDK1 ubiquitination and degradation in these cells ${ }^{24}$.

\section{Conclusion}

Since its in initial discovery as a proto-oncogene, the serine /threonine kinase Akt (also known as PKB) has become a major focus of attention because of its pivotal and critical role in regulating diverse cellular functions as well as is one of the most common pathways leading to human malignancy. The role of PI3K-PDK-AKT signaling pathway in gynecological malignancy seems to play a pivotal role, but it necessitates further investigation, which could lead to novel therapeutic strategies of eventual advantages to many cancer patients.

\section{References}

1. Dieterle AM, Böhler P, Keppeler H, Alers S, Berleth N, Drießen S, Hieke N, Pietkiewicz S, Löffler AS, Peter C, Gray A, Leslie NR, Shinohara H, Kurosaki T, Engelke M, Wienands J, Bonin M, Wesselborg S, Stork B. PDK1 controls upstream PI3K expression and PIP3 generation. Oncogene. 2014 Jun 5;33(23):3043-53.

2. Wu YH, Chang TH, Huang YF, Chen CC, Chou CY. COL11A1 confers chemoresistance on ovarian cancer cells through the activation of Akt/c/ EBP $\beta$ pathway and PDK1 stabilization. Oncotarget. 2015 Sep 15;6(27):23748-63.

3. Mirza AM, Gysin S, Malek N, Nakayama K, Roberts JM, McMahon M. Cooperative regulation of the cell division cycle by the protein kinases RAF and AKT. Mol Cell Biol. 2004 Dec; 24(24):10868-81. PubMed PMID: 15572689; PubMed Central PMCID: PMC533961.

4. Markowska A, Pawałowska M, Lubin J, Markowska J. Signalling pathways in endometrial cancer. Contemp Oncol (Pozn). 2014;18(3):143-8.

5. Faes S, Dormond O. PI3K and AKT: Unfaithful Partners in Cancer. Int J Mol Sci. 2015 Sep 3;16(9):21138-52.

6. Cheaib B, Auguste A, Leary A. The PI3K/Akt/ mTOR pathway in ovarian cancer: therapeutic opportunities and challenges. Chin J Cancer. 2015 Jan;34(1):4-16.

7. Bruhn MA, Pearson RB, Hannan RD, Sheppard 
KE. AKT-independent PI3-K signaling in cancer emerging role for SGK3. Cancer Manag Res. 2013 Aug 26; 5:281-92.

8. Cheng JQ, Godwin AK, Bellacosa A, Taguchi T, Franke TF, Hamilton TC, Tsichlis PN, Testa JR. AKT2, a putative oncogene encoding a member of a subfamily of protein-serine/threonine kinases, is amplified in human ovarian carcinomas. Proc Natl Acad Sci U S A. 1992 Oct 1;89(19):9267-71.

9. Arranz E, Robledo M, Martínez B, Gallego J, Román A, Rivas C, Benítez J. Incidence of homogeneously staining regions in non-Hodgkin lymphomas. Cancer Genet Cytogenet. 1996 Mar;87(1):1-3.

10. Cheng JQ, Ruggeri B, Klein WM, Sonoda G, Altomare DA, Watson DK, Testa JR. Amplification of AKT2 in human pancreatic cells and inhibition of AKT2 expression and tumorigenicity by antisense RNA. Proc Natl Acad Sci U S A. 1996 Apr 16;93(8):3636-41.

11. Miwa W, Yasuda J, Murakami Y, Yashima K, Sugano K, Sekine T, Kono A, Egawa S, Yamaguchi K, Hayashizaki Y, Sekiya T. Isolation of DNA sequences amplified at chromosome 19q13.1-q13.2 including the AKT2 locus in human pancreatic cancer. Biochem Biophys Res Commun. 1996 Aug 23;225(3):968-74.

12. Ruggeri BA, Huang L, Wood M, Cheng JQ, Testa JR. Amplification and overexpression of the AKT2 oncogene in a subset of human pancreatic ductal adenocarcinomas. Mol Carcinog. 1998 Feb;21(2):81-6.

13. Xu X, Sakon M, Nagano H, Hiraoka N, Yamamoto $H$, Hayashi N, Dono K, Nakamori S, Umeshita K, Ito Y, Matsuura N, Monden M. Akt2 expression correlates with prognosis of human hepatocellular carcinoma. Oncol Rep. 2004 Jan;11(1):25-32.

14. Staal SP. Molecular cloning of the Akt oncogene and its human homologues AKT1 and AKT2: amplification of AKT1 in a primary human gastric adenocarcinoma. Proc Natl Acad Sci U S A. 1987
Jul;84(14):5034-7.

15. Knobbe CB, Reifenberger G. Genetic alterations and aberrant expression of genes related to the phosphatidyl-inositol-3'-kinase/protein kinase B (Akt) signal transduction pathway in glioblastomas. Brain Pathol. 2003 Oct;13(4):507-18.

16. Nakatani K, Thompson DA, Barthel A, Sakaue H, Liu W, Weigel RJ, Roth RA. Up-regulation of Akt3 in estrogen receptor-deficient breast cancers and androgen-independent prostate cancer lines. J Biol Chem. 1999 Jul 30;274(31):21528-32.

17. Sun M, Wang G, Paciga JE, Feldman RI, Yuan ZQ, Ma XL, Shelley SA, Jove R, Tsichlis PN, Nicosia SV, Cheng JQ. AKT1/PKB alpha kinase is frequently elevated in human cancers and its constitutive activation is required for oncogenic transformation in NIH3T3 cells. Am J Pathol. 2001 Aug;159(2):431-7.

18. Yuan ZQ Sun M, Feldman RI, Wang G, Ma X, Jiang C, Coppola D, Nicosia SV, Cheng JQ. Frequent activation of AKT2 and induction of apoptosis by inhibition of phosphoinositide-3-OH kinase/Akt pathway in human ovarian cancer. Oncogene. 2000 May 4;19(19):2324-30.

19. Altomare DA, Tanno S, De Rienzo A, KleinSzanto AJ, Tanno S, Skele KL, Hoffman JP, Testa JR. Frequent activation of AKT2 kinase in human pancreatic carcinomas. J Cell Biochem. 2002;87(4):470-6. Erratum in: J Cell Biochem. 2003 Jan 1;88(1):470.

20. Zhu J, Li Y, Guan C, Chen Z. Anti-proliferative and pro-apoptotic effects of 3,3'-diindolylmethane in human cervical cancer cells. Oncol Rep. 2012 Sep;28(3):1063-8.

21. Bertelsen BI, Steine SJ, Sandvei R, Molven A, Laerum OD. Molecular analysis of the PI3K-AKT pathway in uterine cervical neoplasia: frequent PIK3CA amplification and AKT phosphorylation. Int J Cancer. 2006 Apr 15;118(8):1877-83.

22. Mori N, Kyo S, Sakaguchi J, Mizumoto Y, Ohno S, 
Maida Y, Hashimoto M, Takakura M, Inoue M. Concomitant activation of AKT with extracellular-regulated kinase $1 / 2$ occurs independently of PTEN or PIK3CA mutations in endometrial cancer and may be associated with favorable prognosis. Cancer Sci. 2007 Dec;98(12):1881-8.

23. Merritt MA, Cramer DW. Molecular pathogenesis of endometrial and ovarian cancer. Cancer Biomark. 2010;9(1-6):287-305.

24. Longuespée R, Boyon C, Desmons A, Vinatier D,
Leblanc E, Farré I, Wisztorski M, Ly K, D’Anjou F, Day R, Fournier I, Salzet M. Ovarian cancer molecular pathology. Cancer Metastasis Rev. 2012 Dec;31(3-4):713-32.
Received 29-7-2019

Revised 3-9-2019

Accepted 16-9-2019 